\title{
Estudio de la adición de agentes oxidantes y gas de arrastre para la caracterización del tamaño de nanopartículas de oro mediante espectroscopia de absorción atómica de fuente continua con atomización electrotérmica
}

\author{
Raúl Garde, Martín Resano, Esperanza García \\ Afiliación: Grupo de Métodos Analíticos Rápidos mediante Técnicas Espectroscópicas (MARTE) \\ Instituto de Investigación en Ingeniería de Aragón (I3A) \\ Departamento de Química Analítica, Universidad de Zaragoza, Pedro Cerbuna 12, 50009, Zaragoza, Spain. \\ Tel. 843510-, e-mail: 612737@unizar.es
}

\begin{abstract}
En estudios anteriores, mediante la aplicación de una rampa de atomización lenta $\left(150^{\circ} \mathrm{C} \mathrm{s}^{-1}\right)$, ha sido posible la caracterización del tamaño medio de nanopartículas de oro (AuNPs) mediante espectroscopia de absorción atómica de fuente contínua de alta resolución con atomización electrotérmica (HR CS GFAAS) sin utilización de modificantes de matriz o etapas previas. El parámetro ultilizado para este fin es el tiempo del máximo de aparición de la señal, también llamado atomization delay, que se puede correlacionar con el tamaño de NP. Sin embargo, la baja reproducibilidad de las señales de Au (III) y su tendencia a formar picos bimodales son aspectos mejorables del método planteado. En este estudio se evaluó la introducción de agentes oxidantes y/o de un gas de arrastre para mejorar la reproducibilidad del método.
\end{abstract}

\section{Introducción}

La nanotecnología es uno de los campos de mayor desarrollo en los últimos años para diversas aplicaciones como sensores, medicina o ciencia de materiales por su relación superficie-volumen y sus propiedades derivadas de esta característica. Es por ello que el desarrollo de metodologías que aúnen la posibilidad de cuantificar y caracterizar la muestra al mismo tiempo es de gran interés.

Una posible alternativa a las ya existentes es la espectroscopia de absorción atómica de fuente continua de alta resolución con atomización electrotérmica (HR CS GFAAS), ya que estudios iniciales permitieron diferenciar entre iones plata y nanopartículas de este elemento [1] [2], debido a la diferente temperatura de atomización entre estas dos especies.
El objetivo de este estudio es evaluar las capacidades de la técnica para caracterizar el tamaño medio de NPs de oro en suspensión en presencia de un agente oxidante y gas de arrastre durante la atomización para evitar picos bimodales y obtener así señales de Au (III) comparables a las de NPs.

\section{Experimental y Resultados}

Todos los experimentos se llevaron a cabo en un espectrómetro de absorción atómica de fuente continua (contrAA 700, Analytik Jena, Jena, Alemania), equipado con un atomizador de grafito de calentamiento transversal. Las muestras de nanopartículas de oro en suspensión de tamaños 5 , $20, \quad 50, \quad 80$ y $100 \mathrm{~nm}$ se obtuvieron de Nanocomposix (Praga, República Checa). Agua purificada obtenida de un sistema Mili-Q se utilizaron para diluir las muestras a la concentración deseada para su posterior análisis previa sonicación de las suspensiones de nanopartículas. $\mathrm{HNO}_{3} 65 \%$ y $\mathrm{H}_{2} \mathrm{O}_{2} \quad 30 \%$ diluídos se utilizaron como agentes oxidantes.

En los estudios previos sobre plata, queda patente la diferente temperatura de atomización dependiendo del tamaño de partícula de la muestra, necesitando mayores temperaturas para atomizar partículas de mayor tamaño. Mediante la optimización de la rampa de atomización y la temperatura de atomización, fue posible obtener una separación entre las señales de oro iónico y AuNP de $50 \mathrm{~nm}$ con una separación de más de 1 segundo. En la tabla 1 se indica el programa optimizado aplicado a lo largo de todos los experimentos.

El análisis de las diferentes nanopartículas mostró como cada una de ellas atomizaba más tarde conforme aumentaba el tamaño de partícula. En la 
figura 1 se representa el tiempo de aparición del máximo de señal frente al tamaño medio medido mediante TEM proporcionado por Nanocomposix. Se puede observar que al representar los diferentes tamaños aparecen dos regiones lineales; una primera de mayor sensibilidad desde los iones de oro hasta las nanopartículas de 20 nanómetros y la segunda de menor sensibilidad desde $20 \mathrm{~nm}$ hasta la muestra de $100 \mathrm{~nm}$. [3]

Sin embargo, esta correlación presenta el problema de que las señales de Au (III) no son unimodales y gaussianas como las nanopartículas, si no que presentan una cola adicional, que se superpone a la región de aparición de NPs. Esto podría deberse a que durante la aplicación del programa de temperaturas se estén formando NPs en proporciones y tamaños variables. Para evitar la aparición de esta cola, se evaluó la utilización de dos agentes oxidantes; $\mathrm{HNO}_{3}$ y $\mathrm{H}_{2} \mathrm{O}_{2}$ en proporciones diferentes y además la introducción de una corriente de Ar durante la etapa de atomización.

La adición de ambos agentes oxidantes disminuyó la intensidad del hombro, pero no consiguió eliminarlo completamente, se decidió emplear $\mathrm{HNO}_{3}$ debido a la mayor estabilidad durante las sesiones experimentales, además, se observó que al incrementar la cantidad de oxidante, las NPs de 5 $\mathrm{nm}$ aceleraban su atomización, apareciendo a tiempos de atomización correspondientes a $\mathrm{Au}$ (III), las NPs mas grandes no experimentaron oxidación. La combinación de agente oxidante y la introducción de una corriente de $\mathrm{Ar}$ de arrastre durante la atomización, sí que consiguió obtener señales reproducibles y unimodales para todas las partículas manteniendo la separación obtenida

\begin{tabular}{ccccc}
\hline $\begin{array}{c}\text { Programa de } \\
\text { Eemperatura }\end{array}$ & $\begin{array}{c}\text { Temperatura } \\
/{ }^{\circ} \mathrm{C} \mathrm{s}^{-1}\end{array}$ & $\begin{array}{c}\text { Rampa } \\
/{ }^{\circ} \mathrm{C} \mathrm{s}^{-1}\end{array}$ & $\begin{array}{c}\text { Tiempo de } \\
\text { espera } / \mathrm{s}\end{array}$ & $\begin{array}{c}\text { Flujo de gas } \\
\mathrm{Ar} / \mathrm{L} \mathrm{min}^{-1}\end{array}$ \\
\hline Secado & 90 & 3 & 20 & 2 \\
Secado & 110 & 5 & 10 & 2 \\
Pirólisis & 500 & 50 & 20 & 2 \\
& & & & \\
Pirólisis & 500 & 0 & 10 & 2 \\
Atomización & 2200 & 150 & 4 & $0-0.1^{\mathrm{a}}$ \\
Limpieza & 2450 & 500 & 4 & 2 \\
\hline alujo de gas Ar en los experimentos con gas de arrastre
\end{tabular}

Tabla 1. Programa optimizado utilizado. mediante el método estándar entre las señales de NPs con la desventaja de pérdida de sensibilidad de un $60 \%$ debida principalmente a la corriente de Ar, evitando que la señal se produzca por difusión exclusivamente. Esta combinación de oxidación y arrastre evita que se produzca la deposición y reducción en los sitios activos del carbono, que podrían actuar como centros de nucleación para la formación in situ de NPs durante la etapa de atomización, consiguiendo una mejor reproducibilidad del método y mayor facilidad para modelizar las señales y poder empezar a evaluar el método en muestras de interés biológico.

\section{Conclusiones}

La aplicación de rampas de atomización lentas permite la caracterización de tamaño de nanopartículas de oro mediante correlación logarítmica, pero presenta las dificultades de irreproducibilidad al monitorizar Au (III) y NPs de menor tamaño. Sin embargo, la combinación de Ar como gas de arrastre durante la atomización y la utilización de $\mathrm{HNO}_{3}$ diluído a una concentración de XX M previamente optimizada como agente oxidante permitió obtener señales unimodales y mantener la separación de las señales sacrificando un $60 \%$ de sensibilidad.

\section{REFERENCIAS}

[1]. F. Gagné, P. Turcotte and C. Gagnon, Anal. Bioanal. Chem., 2012, 404, 2067-2072.

[2]. N. S. Feichtmeier and K. Leopold, Anal. Bioanal. Chem., 2014, 406, 3887-3894.

[3]. M. Resano, E. Garcia-Ruiz and R. Garde, $J$. Anal. At. Spectrom., 2016, 31, 2233-2241.

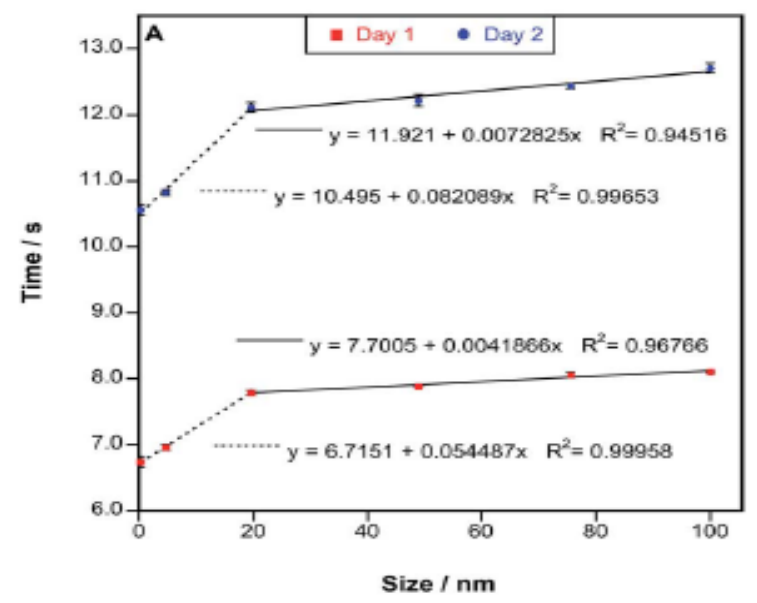

Fig. 1. Atomization delay frente al tamaño de partícula. 